\title{
Up- and Down-operators on Young's Lattice
}

\author{
Ricky Ini Liu* Christian Smith \\ Department of Mathematics \\ North Carolina State University \\ Raleigh, NC, U.S.A. \\ $\{$ riliu, casmit34\}@ncsu.edu
}

Submitted: Dec 18, 2020; Accepted: Jul 20, 2021; Published: Jul 30, 2021

(C) The authors. Released under the CC BY-ND license (International 4.0).

\begin{abstract}
The up-operators $u_{i}$ and down-operators $d_{i}$ (introduced as Schur operators by Fomin) act on partitions by adding/removing a box to/from the $i$ th column if possible. It is well known that the $u_{i}$ alone satisfy the relations of the (local) plactic monoid, and the present authors recently showed that relations of degree at most 4 suffice to describe all relations between the up-operators. Here we characterize the algebra generated by the up- and down-operators together, showing that it can be presented using only quadratic relations.
\end{abstract}

Mathematics Subject Classifications: 05E18, 06A07

\section{Introduction}

The up-operators $u_{i}$ for $i \in \mathbf{N}$ act on a partition $\lambda$ by adding a box to the $i$ th column of $\lambda$ if the result is a partition and by sending $\lambda$ to 0 otherwise. Similarly, the down-operators $d_{i}$ act on $\lambda$ by subtracting a box from the $i$ th column if the result is a partition and by sending it to 0 otherwise. These operators were introduced as Schur operators by Fomin [1] and further discussed by Fomin and Greene [2] in the context of noncommutative Schur functions. They can also be seen as refinements of the raising and lowering operators $U$ and $D$ acting on Young's lattice as defined by Stanley [7] in his study of differential posets.

${ }^{*}$ R. I. Liu and C. Smith were partially supported by National Science Foundation grant DMS-1700302. R. I. Liu was also partially supported by National Science Foundation grant CCF-1900460. 
It was noted in [2] that the $u_{i}$ give a representation for the local plactic monoid as they satisfy the relations:

$$
\begin{aligned}
u_{i} u_{j} & =u_{j} u_{i} \quad \text { for }|i-j| \geqslant 2, \\
u_{i} u_{i+1} u_{i} & =u_{i+1} u_{i} u_{i}, \\
u_{i+1} u_{i} u_{i+1} & =u_{i+1} u_{i+1} u_{i} .
\end{aligned}
$$

(In particular, the $u_{i}$ satisfy the classical Knuth relations of the plactic monoid - see for instance [4].) The current authors proved in [5] (see also Meinel [6]) that the $u_{i}$ also satisfy the additional degree 4 relation

$$
u_{i+1} u_{i+2} u_{i+1} u_{i}=u_{i+1} u_{i+2} u_{i} u_{i+1}
$$

and that this relation along with the local plactic relations characterize the algebra generated by the $u_{i}$, therein called the algebra of Schur operators.

It was also noted in [1] (using the fact that the down-operators can be thought of as transposes of the up-operators) that the $d_{i}$ satisfy:

$$
\begin{aligned}
d_{j} d_{i} & =d_{i} d_{j} \quad \quad \text { for }|i-j| \geqslant 2, \\
d_{i} d_{i+1} d_{i} & =d_{i} d_{i} d_{i+1}, \\
d_{i+1} d_{i} d_{i+1} & =d_{i} d_{i+1} d_{i+1},
\end{aligned}
$$

and that together the $u_{i}$ and $d_{i}$ satisfy:

$$
\begin{aligned}
d_{i} u_{j} & =u_{j} d_{i} \quad \text { for } i \neq j, \\
d_{1} u_{1} & =i d \\
d_{i+1} u_{i+1} & =u_{i} d_{i} .
\end{aligned}
$$

In this paper we give a complete description of the algebra generated by the $u_{i}$ and $d_{i}$, which we call the algebra of up- and down-operators for Young's lattice. Surprisingly, the following theorem shows that quadratic relations suffice to give a presentation of this algebra.

Theorem 1. The algebra of up- and down-operators for Young's lattice is defined by the relations:

$$
\begin{aligned}
u_{i} u_{j} & =u_{j} u_{i} & & \text { for }|i-j| \geqslant 2, \\
d_{i} d_{j} & =d_{j} d_{i} & & \text { for }|i-j| \geqslant 2, \\
d_{i} u_{j} & =u_{j} d_{i} & & \text { for } i \neq j, \\
d_{1} u_{1} & =i d, & & \\
d_{i+1} u_{i+1} & =u_{i} d_{i} . & &
\end{aligned}
$$

In particular, it follows that the local plactic relations are implied by the quadratic relations in Theorem 1. 
In contrast, we also give a complete description of the subalgebra generated by $u_{t}$ and $d_{t}$ for a fixed $t>1$ and show that it cannot be presented using relations of bounded degree.

We provide necessary background information about partitions and the up- and downoperators in Section 2. The characterization of the algebra of up- and down- operators is given in Section 3, and a discussion of subalgebras can be found in Section 4.

\section{Preliminaries}

In this section, we discuss some background on partitions and up- and down-operators.

\subsection{Partitions}

A partition $\lambda=\left(\lambda_{1}, \lambda_{2}, \ldots\right)$ of $|\lambda|=\sum_{i=1} \lambda_{i}$ is a sequence of nonincreasing nonnegative integers. We associate to each partition a collection of left-aligned boxes with $\lambda_{i}$ boxes in the $i$ th row called the Young diagram of $\lambda$. We define the conjugate partition $\lambda^{\prime}$ to be the partition whose Young diagram is obtained by reflecting the Young diagram of $\lambda$ across the main diagonal.

We consider the partial order on partitions $\lambda$ and $\mu$ such that $\mu \leqslant \lambda$ if and only if the Young diagram of $\mu$ fits inside the Young diagram of $\lambda$, that is, $\mu_{i} \leqslant \lambda_{i}$ for all $i$. Note that this means that if $\mu \leqslant \lambda$, then $\lambda$ covers $\mu$ (denoted $\mu \lessdot \lambda$ ) if and only if $\lambda / \mu$ is a single box, where $\lambda / \mu$ is the skew Young diagram consisting of all boxes in $\lambda$ that are not in $\mu$. We take Young's lattice $(\mathbf{Y}, \leqslant)$ to be the partially ordered set of partitions with the above partial order.

\subsection{Words in the alphabet}

Let $\mathbf{N}=\{1,2, \ldots\}, \overline{\mathbf{N}}=\{\overline{1}, \overline{2}, \ldots\}$, and $\Gamma=\mathbf{N} \cup \overline{\mathbf{N}}$. We refer to elements $1,2, \ldots$ of $\mathbf{N}$ as unbarred letters and elements $\overline{1}, \overline{2}, \ldots$ of $\overline{\mathbf{N}}$ as barred letters.

Let $x=x_{1} \cdots x_{\ell}$ be a word of length $\ell$ in the alphabet $\Gamma$. The weight of $x$ is the vector $w(x)=\left(w_{1}(x), w_{2}(x), \ldots\right)$ where

$$
w_{i}(x)=(\text { the number of times } i \text { appears in } x)-(\text { the number of times } \bar{i} \text { appears in } x) \text {. }
$$

We also define the $\alpha$-vector of $x$ to be $\alpha(x)=\left(\alpha_{1}(x), \alpha_{2}(x), \ldots\right)$ where

$$
\alpha_{i}(x)=\max \left\{w_{i+1}(\tilde{x})-w_{i}(\tilde{x}) \mid \tilde{x} \text { is a suffix subword of } x\right\} .
$$

Here a suffix subword $\tilde{x}$ is a word of the form $\tilde{x}=x_{j} x_{j+1} \cdots x_{\ell}$ for some $1 \leqslant j \leqslant \ell+1$. When $j=\ell+1, \tilde{x}$ is the empty word, in which case $w_{i+1}(\tilde{x})=w_{i}(\tilde{x})=0$, so it follows that $\alpha_{i}(x) \geqslant 0$ for all $i$.

Example 2. Let $x=11 \overline{332} 32 \overline{1} 21$. Then we have $w(x)=(2,1,-1,0, \ldots)$ and $\alpha(x)=$ $(2,0,1,0, \ldots)$. For instance, for $\alpha_{1}(x)=2$, the maximum value of $w_{2}(\tilde{x})-w_{1}(\tilde{x})$ first occurs when $\tilde{x}=2 \overline{1} 21$. 


\section{$2.3 \quad$ Up-operators and down-operators}

Let $\mathcal{U}$ be the free associative algebra over the complex field $\mathbf{C}$ generated by elements $u_{i}$ for $i \in \Gamma$. We will write $d_{i}=u_{\bar{i}}$ for all barred letters $\bar{i}$. For any word $x=x_{1} x_{2} \ldots x_{\ell}$ in the alphabet $\Gamma$, we define $u_{x}=u_{x_{1}} u_{x_{2}} \cdots u_{x_{\ell}}$. We also use the alternate notation $\mathrm{i}=u_{i}$ for $i \in \Gamma$. To avoid potential confusion in the future, we note now that $(\mathrm{i}+\mathrm{j})$ denotes $u_{i+j}$ and not the sum $u_{i}+u_{j}$.

Let $\mathbf{C}[\mathbf{Y}]$ be the complex vector space with basis $\mathbf{Y}$. We define an action of $\mathcal{U}$ on $\mathbf{C}[\mathbf{Y}]$ in the following way. For $\lambda \in \mathbf{Y}$ and $i \in \mathbf{N}$, we let

$$
u_{i}(\lambda)= \begin{cases}\mu & \text { if } \mu \in \mathbf{Y} \text { and } \mu / \lambda \text { is a single box in column } i \\ 0 & \text { otherwise }\end{cases}
$$

and

$$
d_{i}(\lambda)= \begin{cases}\mu & \text { if } \mu \in \mathbf{Y} \text { and } \lambda / \mu \text { is a single box in column } i, \\ 0 & \text { otherwise. }\end{cases}
$$

Example 3. Let $\lambda=(3,1)$. Then $u_{2}(\lambda)=(3,2), d_{3} u_{2}(\lambda)=(2,2)$, but $d_{1} d_{3} u_{2}(\lambda)=0$ since subtracting a box from the first column does not yield a partition.

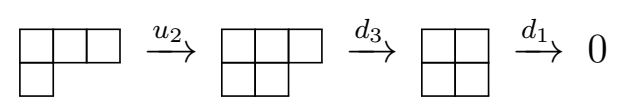

Note that $u_{i}(\lambda)$ is either 0 or a partition that covers $\lambda$ in $\mathbf{Y}$, so we refer to $u_{i}$ as an up-operator, and similarly we call $d_{i}$ a down-operator. These operators were introduced by Fomin [1] under the name Schur operators.

The action of $u_{x}$ on partitions is determined by the weight and $\alpha$-vector of $x$ as follows.

Proposition 4. Let $x$ be a word and $\lambda \in \mathbf{Y}$. Then

$$
u_{x}(\lambda)= \begin{cases}\left(\lambda_{1}^{\prime}+w_{1}(x), \lambda_{2}^{\prime}+w_{2}(x), \ldots\right)^{\prime} & \text { if } \lambda_{i}^{\prime}-\lambda_{i+1}^{\prime} \geqslant \alpha_{i}(x) \text { for all } i \\ 0 & \text { otherwise }\end{cases}
$$

Proof. We have $u_{x}(\lambda) \neq 0$ if and only if $u_{\tilde{x}}(\lambda)$ is a partition for each suffix subword $\tilde{x}$ of $x$. Fix some $\tilde{x}$ and suppose $\mu=u_{\tilde{x}}(\lambda) \neq 0$. We then have $\mu_{i}^{\prime}=\lambda_{i}^{\prime}+w_{i}(\tilde{x})$ for all $i$. The condition for $\mu$ to be a partition is that $\mu_{i}^{\prime} \geqslant \mu_{i+1}^{\prime}$ for all $i$, or equivalently

$$
\lambda_{i}^{\prime}+w_{i}(\tilde{x}) \geqslant \lambda_{i+1}^{\prime}+w_{i+1}(\tilde{x}) .
$$

Rearranging this gives

$$
\lambda_{i}^{\prime}-\lambda_{i+1}^{\prime} \geqslant w_{i+1}(\tilde{x})-w_{i}(\tilde{x}) .
$$

By the definition of $\alpha_{i}(x)$, these inequalities hold for all suffix subwords $\tilde{x}$ if and only if $\lambda_{i}^{\prime}-\lambda_{i+1}^{\prime} \geqslant \alpha_{i}(x)$.

The following corollary then follows from Proposition 4. 
Corollary 5. Let $x$ and $y$ be words. Then $u_{x}$ and $u_{y}$ act identically on $\mathbf{Y}$ if and only if $\alpha(x)=\alpha(y)$ and $w(x)=w(y)$.

Proof. The backwards implication is immediate from Proposition 4. For the forward direction, suppose $\alpha(x) \neq \alpha(y)$. Then we may assume without loss of generality that $\alpha_{j}(x)<\alpha_{j}(y)$ for some $j$. Taking $\lambda$ such that $\lambda_{i}^{\prime}-\lambda_{i+1}^{\prime}=\alpha_{i}(x)$, we have $u_{x}(\lambda) \neq 0=$ $u_{y}(\lambda)$, so $u_{x}$ and $u_{y}$ do not act identically. If instead $\alpha(x)=\alpha(y)$ but $w(x) \neq w(y)$, then for this same choice of $\lambda, u_{x}(\lambda) \neq u_{y}(\lambda)$ by Proposition 4 .

It was noted in [1] that $u_{i}$ and $d_{i}$ are transposes with respect to the basis $\mathbf{Y}$, which we may write as $u_{i}^{T}=d_{i}$. Also in [1], various relations among the $u_{i}$ and $d_{i}$ were described, including the local plactic relations and various quadratic relations (as described in Section 3). Our main result will be to show that in fact these quadratic relations generate all relations between these operators.

\section{The algebra of up- and down-operators}

Let $I$ be the two-sided ideal consisting of all elements of $\mathcal{U}$ that annihilate $\mathbf{C}[\mathbf{Y}]$. We call the algebra $\mathcal{U} / I$ the algebra of up-and down-operators for Young's lattice. Let $J$ be the two-sided ideal generated by the following relations.

$$
\begin{array}{rlrl}
u_{i} u_{j} & \equiv u_{j} u_{i} & & \text { for }|i-j| \geqslant 2, \\
d_{i} d_{j} & \equiv d_{j} d_{i} & & \text { for }|i-j| \geqslant 2, \\
d_{i} u_{j} & \equiv u_{j} d_{i} & & \text { for } i \neq j, \\
d_{1} u_{1} & \equiv i d, & \\
d_{i+1} u_{i+1} & \equiv u_{i} d_{i} . &
\end{array}
$$

Our main result (Theorem 1) will be to show that $I=J$. We first verify that $J \subseteq I$.

Proposition 6. The inclusion of ideals $J \subseteq I$ holds.

Proof. It suffices to show that for each of (1)-(5), the two terms in the relation are in fact equivalent modulo $I$. We show this for relation (5); the other relations are similar. By Corollary 5 we need only show $\alpha(x)=\alpha(y)$ and $w(x)=w(y)$ where $x=i \bar{i}$ and $y=$ $\overline{(i+1)}(i+1)$. Indeed, $w(x)=(0,0, \ldots)=w(y)$, while $\alpha(x)=(0, \ldots, 0,1,0, \ldots)=\alpha(y)$, where the 1 occurs in the $i$ th position.

It therefore remains only to show that $I \subseteq J$. The next proposition proves that $I$ is a binomial ideal, that is, $I$ is generated by elements of the form $u_{x}-u_{y}$. The proof of this proposition is very similar to that of Proposition 3.3 in [5], but we include it here for completeness.

Proposition 7. The ideal I is a binomial ideal. 
Proof. Let $I^{\prime}$ be the two-sided ideal generated by all binomials $u_{x}-u_{y}$ such that $u_{x} \equiv u_{y}$ $(\bmod I)$, and suppose $R \in I$. Since $\mathcal{U}$ is graded by weight and $I$ is homogeneous with respect to weight, we may assume that all terms appearing in $R$ have weight $w$ for some $w=\left(w_{1}, w_{2}, \ldots\right)$. We can then find $R^{\prime} \equiv R\left(\bmod I^{\prime}\right)$ for some

$$
R^{\prime}=\sum_{i=1}^{n} c_{x^{(i)}} u_{x^{(i)}},
$$

where $x^{(i)}$ is a word in $\Gamma$ of weight $w, u_{x^{(i)}} \not \equiv u_{x^{(j)}}(\bmod I)$ whenever $i \neq j$, and $0 \neq$ $c_{x^{(i)}} \in \mathbf{C}$ for all $i \in[n]$. In particular, by Corollary 5 , the $\alpha\left(x^{(i)}\right)$ are distinct, so suppose without loss of generality that they occur in lexicographic order.

If $n \geqslant 1$, let $\lambda \in \mathbf{Y}$ be such that $\lambda_{k}^{\prime}-\lambda_{k+1}^{\prime}=\alpha_{k}\left(x^{(1)}\right)$ for all $k$. By Proposition 4 , $u_{x^{(1)}}(\lambda) \neq 0$. For each $i>1$, by the lexicographic ordering, there exists some $s$ such that $\alpha_{s}\left(x^{(1)}\right)<\alpha_{s}\left(x^{(i)}\right)$. Then by Proposition $4, u_{x^{(i)}}(\lambda)=0$. Thus $0=R^{\prime}(\lambda)=c_{x^{(1)}} u_{x^{(1)}}(\lambda)$, which implies $c_{x^{(1)}}=0$. This is a contradiction, so we must have $R^{\prime}=0$. Thus $I=I^{\prime}$.

Our goal for the rest of this section is to show that if $u_{x} \equiv u_{y}(\bmod I)$, then $u_{x} \equiv u_{y}$ $(\bmod J)$. Our general strategy is as follows. Let $\left[u_{x}\right]_{I}$ be the equivalence class of $u_{x}$ modulo $I$. We will construct a representative word $[x]$ such that $u_{[x]} \in\left[u_{x}\right]_{I}$. This representative will only depend on $\alpha(x)$ and $w(x)$, so if $u_{x} \equiv u_{y}(\bmod I)$, then $[x]=[y]$. We will then show that $u_{x} \equiv u_{[x]}(\bmod J)$ and similarly for $y$, which will complete the proof.

Definition 8. For a word $x$, define

$$
\begin{aligned}
m(x) & =\max _{i \in \mathbf{N}}\left\{-\left(\alpha_{i}(x)+w_{i}(x)\right)\right\} \geqslant 0, \\
n(x) & =\max \{t \in \mathbf{N} \mid t \text { or } \bar{t} \text { appears in } x\} .
\end{aligned}
$$

For any $m \geqslant m(x), n \geqslant n(x)$, we let

$$
[x]_{m, n}=\left(\overline{1}^{m} \cdots \bar{n}^{m}\right)\left(n^{\beta_{n}^{m}(x)} \bar{n}^{\alpha_{n}(x)} \cdots 1^{\beta_{1}^{m}(x)} \overline{1}^{\alpha_{1}(x)}\right)
$$

where $\beta_{i}^{m}(x)=\alpha_{i}(x)+w_{i}(x)+m$.

Note that the definition of $m$ ensures that all of the exponents appearing in the definition of $[x]_{m, n}$ are nonnegative. We will often abbreviate $[x]=[x]_{m, n}$. We now show that indeed $u_{[x]} \in\left[u_{x}\right]_{I}$.

Proposition 9. For any word $x, u_{x} \equiv u_{[x]}(\bmod I)$.

Proof. Let $i \in \mathbf{N}$. Then $w_{i}([x])=-m+\beta_{i}^{m}(x)-\alpha_{i}(x)=w_{i}(x)$. We now show that $\alpha_{i}([x])=\alpha_{i}(x)$. For ease of notation, we will write $\alpha_{i}=\alpha_{i}(x), w_{i}=w_{i}(x)$, and $\beta_{i}=$ $\beta_{i}^{m}(x)$. Since $\alpha_{i}$ relies only upon the appearances of $i, \bar{i},(i+1)$, and $\overline{(i+1)}$ in $x$, we need only consider the subword

$$
\bar{i}^{m} \overline{(i+1)}^{m}(i+1)^{\beta_{i+1}} \overline{(i+1)}^{\alpha_{i+1}} i^{\beta_{i}} \bar{i}^{\alpha_{i}} .
$$


To calculate $\alpha_{i}([x])$, we need to find the maximum value of $w_{i+1}(\tilde{x})-w_{i}(\tilde{x})$ for each suffix subword $\tilde{x}$. This value only increases when adding an occurrence of $\bar{i}$ or $(i+1)$ to $\tilde{x}$. Thus we need only verify a few choices of $\tilde{x}$ :

$$
\begin{aligned}
& \tilde{x}=\bar{i}^{\alpha_{i}}: \quad w_{i+1}(\tilde{x})-w_{i}(\tilde{x})=\alpha_{i}, \\
& \tilde{x}=(i+1)^{\beta_{i+1}} \overline{(i+1)}^{\alpha_{i+1}} i^{\beta_{i}} \bar{\alpha}^{\alpha_{i}}: \quad w_{i+1}(\tilde{x})-w_{i}(\tilde{x})=\beta_{i+1}-\alpha_{i+1}-\beta_{i}+\alpha_{i} \\
& \tilde{x}=[x]: \quad w_{i+1}(\tilde{x})-w_{i}(\tilde{x})=w_{i+1}-w_{i} .
\end{aligned}
$$

The maximum of these is just $\alpha_{i}$.

We now wish to show that $u_{x} \equiv u_{[x]_{m, n}}(\bmod J)$ for sufficiently large $m$ and $n$. To that end we will make use of the following two lemmas. As a reminder, we will use $\mathrm{i}$ and $\overline{\mathrm{i}}$ to represent $u_{i}$ and $d_{i}$, respectively.

Lemma 10. Let $x=\overline{1} \cdots \bar{n} n \cdots 1$ for any $n \in \mathbf{N}$. Then $u_{x} \equiv i d(\bmod J)$.

Proof. First note the equivalence

$$
\overline{\mathrm{n}} \mathrm{n}(\mathrm{n}-1) \cdots 1 \equiv(\mathrm{n}-1)(\mathrm{n}-2) \cdots 1 \quad(\bmod J)
$$

which holds by repeated application of (5) and a single use of (4). Then

$$
\begin{aligned}
\overline{1} \cdots \overline{\mathrm{n}} \mathrm{\cdots} \cdots 1 & \equiv \overline{1} \cdots \overline{(\mathrm{n}-1)}(\mathrm{n}-1) \cdots 1 \\
& \equiv \overline{1} \cdots \overline{(\mathrm{n}-2)}(\mathrm{n}-2) \cdots 1 \\
& \vdots \\
& \equiv i d
\end{aligned}
$$

by repeated application of (6).

Lemma 11. The following equivalences hold modulo $J$ :

$$
\begin{aligned}
u_{i} & \equiv u_{i} d_{i} u_{i}, \\
d_{i} & \equiv d_{i} u_{i} d_{i}, \\
u_{i} u_{i+1} u_{i} & \equiv u_{i+1} u_{i} u_{i}, \\
d_{i} d_{i+1} d_{i} & \equiv d_{i} d_{i} d_{i+1}, \\
u_{i+1} u_{i} u_{i+1} & \equiv u_{i+1} u_{i+1} u_{i}, \\
d_{i+1} d_{i} d_{i+1} & \equiv d_{i} d_{i+1} d_{i+1} .
\end{aligned}
$$

Proof. For (7), we have

$$
\begin{aligned}
\mathrm{n} & \equiv \mathrm{n} \overline{1} \cdots \overline{\mathrm{n}} \cdots 1 \\
& \equiv \overline{1} \cdots \overline{(\mathrm{n}-1)} \mathrm{n} \overline{\mathrm{n}} \cdots 1 \\
& \equiv \overline{1} \cdots \overline{(\mathrm{n}-1)} \overline{(\mathrm{n}+1)}(\mathrm{n}+1) \mathrm{n} \cdots 1 \\
& \equiv \overline{(\mathrm{n}+1)}(\mathrm{n}+1) \mathrm{n} \overline{1} \cdots \overline{(\mathrm{n}-1)}(\mathrm{n}-1) \cdots 1 \\
& \equiv \overline{(\mathrm{n}+1)}(\mathrm{n}+1) \mathrm{n} \\
& \equiv \mathrm{n} \bar{n} \mathrm{n} .
\end{aligned}
$$


For (9) we have

$$
\begin{aligned}
\mathrm{n}(\mathrm{n}+1) \mathrm{n} & \equiv \mathrm{n}(\mathrm{n}+1) \overline{(\mathrm{n}+1)}(\mathrm{n}+1) \mathrm{n} \\
& \equiv \mathrm{n} \overline{(\mathrm{n}+2)}(\mathrm{n}+2)(\mathrm{n}+1) \mathrm{n} \\
& \equiv \overline{(\mathrm{n}+2)}(\mathrm{n}+2) \mathrm{n}(\mathrm{n}+1) \mathrm{n} \\
& \equiv(\mathrm{n}+1) \overline{(\mathrm{n}+1)} \mathrm{n}(\mathrm{n}+1) \mathrm{n} \\
& \equiv(\mathrm{n}+1) \mathrm{n} \overline{(\mathrm{n}+1)}(\mathrm{n}+1) \mathrm{n} \\
& \equiv(\mathrm{n}+1) \mathrm{nn} \bar{n} \\
& \equiv(\mathrm{n}+1) \mathrm{nn} .
\end{aligned}
$$

For (11), assume $n \geqslant 2$ (the case when $n=1$ is similar). Then

$$
\begin{aligned}
(\mathrm{n}+1) \mathrm{n}(\mathrm{n}+1) & \equiv(\mathrm{n}+1) \mathrm{n} \bar{n} \mathrm{n}(\mathrm{n}+1) \\
& \equiv(\mathrm{n}+1) \mathrm{n}(\mathrm{n}-1) \overline{(\mathrm{n}-1)}(\mathrm{n}+1) \\
& \equiv(\mathrm{n}+1) \mathrm{n}(\mathrm{n}+1)(\mathrm{n}-1) \overline{(\mathrm{n}-1)} \\
& \equiv(\mathrm{n}+1) \mathrm{n}(\mathrm{n}+1) \overline{\mathrm{n}} \mathrm{n} \\
& \equiv(\mathrm{n}+1) \mathrm{n} \overline{\mathrm{n}}(\mathrm{n}+1) \mathrm{n} \\
& \equiv(\mathrm{n}+1) \overline{(\mathrm{n}+1)}(\mathrm{n}+1)(\mathrm{n}+1) \mathrm{n} \\
& \equiv(\mathrm{n}+1)(\mathrm{n}+1) \mathrm{n} .
\end{aligned}
$$

The proofs of (8), (10), and (12) are similar to the proofs of (7), (9), and (11), respectively.

In particular, one can observe that (9) and (11) are Knuth relations, which, together with (1), verify that the quadratic relations imply that $J$ contains all the relations of the local plactic monoid generated by the $u_{i}$ (see [1]).

We are now ready to prove the heart of our main theorem.

Proposition 12. Let $x=x_{1} \cdots x_{\ell}$ be a word. Then there exist $M, N \in \mathbf{N}$ such that $u_{x} \equiv u_{[x]_{m, n}}(\bmod J)$ for all $m \geqslant M, n \geqslant N$.

Proof. As before, we will abbreviate $[x]=[x]_{m, n}$ and $[y]=[y]_{m, n}$. We proceed by induction on the length of $x$. First suppose $\ell=0$ (that is, $x$ is the empty word), and take any $m, n \geqslant 0$. Then we have $[x]=\overline{1}^{m} \ldots \bar{n}^{m} n^{m} \ldots 1^{m}$ and we wish to show that $u_{[x]} \equiv i d$ $(\bmod J)$. By $(1)$ and $(11)$,

$$
\mathrm{n} \cdots 1 \mathrm{n} \equiv \mathrm{n}(\mathrm{n}-1) \mathrm{n}(\mathrm{n}-2) \cdots 1 \equiv \mathrm{nn}(\mathrm{n}-1) \cdots 1 .
$$

In other words, $\mathrm{n}$ and $\mathrm{n} \cdots 1$ commute. Therefore

$$
\begin{aligned}
(\mathrm{n} \cdots 1) \mathrm{n}^{m-1} \cdots 1^{m-1} & \equiv \mathrm{n}^{m}((\mathrm{n}-1) \cdots 1)(\mathrm{n}-1)^{m-1} \cdots 1^{m-1} \\
& \equiv \mathrm{n}^{m}(\mathrm{n}-1)^{m}((\mathrm{n}-2) \cdots 1)(\mathrm{n}-2)^{m-1} \cdots 1^{m-1} \\
& \vdots \\
& \equiv \mathrm{n}^{m} \cdots 1^{m}
\end{aligned}
$$


so $\mathrm{n}^{m} \cdots 1^{m} \equiv(\mathrm{n} \cdots 1)^{m}$. Similarly by $(2)$ and $(12), \overline{1}^{m} \cdots \overline{\mathrm{n}}^{m} \equiv(\overline{1} \cdots \overline{\mathrm{n}})^{m}$. Then applying Lemma 10 repeatedly to

$$
\overline{1}^{m} \cdots \overline{\mathrm{n}}^{m} \mathrm{n}^{m} \cdots 1^{m} \equiv(\overline{1} \cdots \overline{\mathrm{n}})^{m}(\mathrm{n} \cdots 1)^{m}
$$

gives the claim.

Now suppose the proposition statement is true for all words of length less than $\ell$. Let $x=x_{1} \cdots x_{\ell}$ and $y=x_{1} \cdots x_{\ell-1}$. By induction we know the statement holds for $y$ for some $N^{\prime}, M^{\prime} \in \mathbf{N}$. Then take $M=\max \left\{m(x), M^{\prime}\right\}$ and $N=\max \left\{n(x), N^{\prime}\right\}$ and let $m \geqslant M$ and $n \geqslant N$. By induction we have $u_{x}=u_{y} u_{x_{\ell}} \equiv u_{[y]} u_{x_{\ell}}(\bmod J)$. From this we see that it suffices to show $u_{[y]} u_{x_{\ell}} \equiv u_{[x]}(\bmod J)$. For ease of notation we let $\alpha_{i}=\alpha_{i}(y)$, $\beta_{i}=\beta_{i}^{m}(y), w_{i}=w_{i}(y)$, and $\beta_{i}(x)=\beta_{i}^{m}(x)$ for all $i$.

We now split the argument into four cases depending on $x_{\ell}$ and $\alpha_{i}$. Note that if $x_{\ell}=t$ or $\bar{t}$ for $t \geqslant 1$, then $\alpha_{i}(x)=\alpha_{i}, w_{i}(x)=w_{i}$, and $\beta_{i}(x)=\beta_{i}$ for all $i \neq t, t-1$.

Case 1. Suppose $x_{\ell}=t$ and $\alpha_{t}=0$. We have $\alpha_{t-1}(x)=\alpha_{t-1}+1, w_{t-1}(x)=w_{t-1}$, $\alpha_{t}(x)=0$, and $w_{t}(x)=w_{t}+1$, so that $\beta_{t-1}(x)=\beta_{t-1}+1$ and $\beta_{t}(x)=\beta_{t}+1$. Then

$$
\begin{aligned}
u_{[y]} u_{t} & \equiv \cdots \mathrm{t}^{\beta_{t}}(\mathrm{t}-1)^{\beta_{t-1}} \overline{(\mathrm{t}-1)}^{\alpha_{t-1}} \cdots \mathrm{t} \\
& \equiv \cdots \mathrm{t}^{\beta_{t}}(\mathrm{t}-1)^{\beta_{t-1}} \mathrm{t} \overline{\mathrm{t}-1}^{\alpha_{t-1}} \ldots \\
& \equiv \cdots \mathrm{t}^{\beta_{t}}(\mathrm{t}-1)^{\beta_{t-1}} \mathrm{t} \overline{\mathrm{tt}} \overline{\mathrm{t}}^{\alpha_{t-1}} \ldots \\
& \equiv \cdots \mathrm{t}^{\beta_{t}}(\mathrm{t}-1)^{\beta_{t-1}} \mathrm{t}(\mathrm{t}-1) \overline{(\mathrm{t}-1)}{ }^{\alpha_{t-1}+1} \ldots \\
& \equiv \cdots \mathrm{t}^{\beta_{t}+1}(\mathrm{t}-1)^{\beta_{t-1}+1} \overline{(\mathrm{t}-1)}{ }^{\alpha_{t-1}+1} \ldots \\
& =u_{[x]} .
\end{aligned}
$$

Case 2. Suppose that $x_{\ell}=t$ and $\alpha_{t} \neq 0$. We have $\alpha_{t-1}(x)=\alpha_{t-1}+1, w_{t-1}(x)=w_{t-1}$, $\alpha_{t}(x)=\alpha_{t}-1$, and $w_{t}(x)=w_{t}+1$, so that $\beta_{t-1}(x)=\beta_{t-1}+1$ and $\beta_{t}(x)=\beta_{t}$. Then

$$
\begin{aligned}
& u_{[y]} u_{t} \equiv \cdots \mathrm{t}^{\beta_{t}} \overline{\mathrm{t}}^{\alpha_{t}}(\mathrm{t}-1)^{\beta_{t-1}} \overline{(\mathrm{t}-1)}^{\alpha_{t-1}} \cdots \mathrm{t} \\
& \equiv \cdots \mathrm{t}^{\beta_{t}} \overline{\mathrm{t}}^{\alpha_{t}-1}(\mathrm{t}-1)^{\beta_{t-1}} \overline{\mathrm{t}} \overline{\mathrm{t}} \overline{\mathrm{t}-1}^{\alpha_{t-1}} \cdots
\end{aligned}
$$

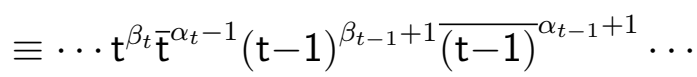

$$
\begin{aligned}
& =u_{[x]} \text {. }
\end{aligned}
$$

Case 3. Suppose that $x_{\ell}=\bar{t}$ and $\alpha_{t-1}=0$. We have $\alpha_{t-1}(x)=0, w_{t-1}(x)=w_{t-1}$, $\alpha_{t}(x)=\alpha_{t}+1$, and $w_{t}(x)=w_{t}-1$, so that $\beta_{t-1}(x)=\beta_{t-1}$ and $\beta_{t}(x)=\beta_{t}$. Then

$$
\begin{aligned}
u_{[y]} d_{t} & \equiv \cdots \mathrm{t}^{\beta_{t}} \overline{\mathrm{t}}^{\alpha_{t}}(\mathrm{t}-1)^{\beta_{t-1}} \cdots \overline{\mathrm{t}} \\
& \equiv \cdots \mathrm{t}^{\beta_{t}} \overline{\mathrm{t}}^{\alpha_{t}+1}(\mathrm{t}-1)^{\beta_{t-1}} \cdots \\
& =u_{[x]} .
\end{aligned}
$$

Case 4. Finally, suppose that $x_{\ell}=\bar{t}$ and $\alpha_{t-1} \neq 0$. We have $\alpha_{t-1}(x)=\alpha_{t-1}-1$, $w_{t-1}(x)=w_{t-1}, \alpha_{t}(x)=\alpha_{t}+1$, and $w_{t}(x)=w_{t}-1$, so that $\beta_{t-1}(x)=\beta_{t-1}-1$ and 
$\beta_{t}(x)=\beta_{t}$. Then

$$
\begin{aligned}
& u_{[y]} d_{t} \equiv \cdots \mathrm{t}^{\beta_{t}} \overline{\mathrm{t}}^{\alpha_{t}}(\mathrm{t}-1)^{\beta_{t-1}} \overline{(\mathrm{t}-1)}^{\alpha_{t-1}} \cdots \overline{\mathrm{t}}
\end{aligned}
$$

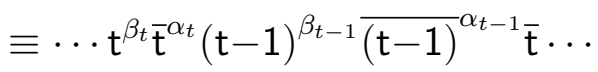

$$
\begin{aligned}
& \equiv \cdots \mathrm{t}^{\beta_{t}} \overline{\mathrm{t}}^{\alpha_{t}}(\mathrm{t}-1)^{\beta_{t-1}-1}(\mathrm{t}-1) \overline{(\mathrm{t}-1)} \overline{\mathrm{t}} \overline{\mathrm{t}-1)}{ }^{\alpha_{t-1}-1} \ldots
\end{aligned}
$$

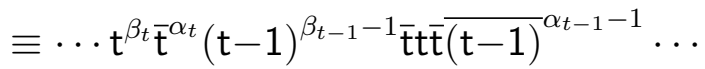

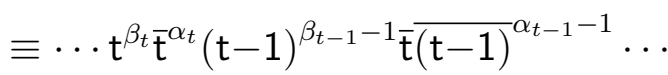

$$
\begin{aligned}
& \equiv \cdots \mathrm{t}^{\beta_{t}} \overline{\mathrm{t}}^{\alpha_{t}+1}(\mathrm{t}-1)^{\beta_{t-1}-1} \overline{(\mathrm{t}-1)}^{\alpha_{t-1}-1} \ldots \\
& =u_{[x]} \text {. }
\end{aligned}
$$

This completes the proof.

It is now easy to complete the proof of our main theorem.

Theorem 13. The ideals $I$ and $J$ are equal. Equivalently, the algebra of up- and downoperators for Young's lattice (generated by the $u_{i}$ and $d_{i}$ ) is determined by relations (1)(5).

Proof. The inclusion $J \subseteq I$ follows from Proposition 7. For the other direction, note that by Proposition 6 we need only prove that $u_{x} \equiv u_{y}(\bmod I)$ implies $u_{x} \equiv u_{y}(\bmod J)$ for words $x$ and $y$. By Proposition 12 there exist nonnegative integers $m$ and $n$ sufficiently large such that $u_{x} \equiv u_{[x]_{m, n}}=u_{[y]_{m, n}} \equiv u_{y}(\bmod J)$.

Remark 14. The up- and down-operators $u_{i}$ and $d_{i}$ refine the raising and lowering operators $U$ and $D$ on Young's lattice introduced by Stanley [7] in his study of differential posets via $U=\sum_{i} u_{i}$ and $D=\sum_{i} d_{i}$. (Although these sums are infinite, only finitely many terms yield a nonzero result when applied to a particular element of $\mathbf{C}[\mathbf{Y}]$.) Therefore the main relation $D U-U D=i d$ defining differential posets must be a consequence of relations (1)-(5). Indeed, we see that

$$
D U-U D=\sum_{i \neq j}\left(d_{i} u_{j}-u_{j} d_{i}\right)+\sum_{i \geqslant 1}\left(d_{i+1} u_{i+1}-u_{i} d_{i}\right)+d_{1} u_{1}=i d .
$$

\section{Subalgebras}

We now turn our attention to various subalgebras generated by up- and down-operators. We briefly discuss a subalgebra studied by the authors in [5], and we introduce two other subalgebras of interest, giving a complete list of relations for each of them.

\subsection{Up-operators and down-operators}

We first consider the subalgebra generated by the up-operators $u_{i}$. Let $\mathcal{U}^{\prime}$ be the subalgebra of $\mathcal{U}$ generated by $u_{i}$ for $i \in \mathbf{N}$. Furthermore, let $I_{\mathcal{U}^{\prime}}=I \cap \mathcal{U}^{\prime}$ be the ideal of 
$\mathcal{U}^{\prime}$ consisting of all elements of $\mathcal{U}^{\prime}$ that annihilate $\mathbf{Y}$. We call $\mathcal{U}^{\prime} / I_{\mathcal{U}^{\prime}}$ the subalgebra of up-operators for Young's lattice. In [5], the present authors described this as the algebra of Schur operators and proved the following theorem. (See also Meinel [6].)

Theorem 15. The ideal $I_{\mathcal{U}^{\prime}}$ is generated by the following relations:

$$
\begin{aligned}
u_{i} u_{j} & \equiv u_{j} u_{i} \quad \text { for }|i-j| \geqslant 2, \\
u_{i} u_{i+1} u_{i} & \equiv u_{i+1} u_{i} u_{i}, \\
u_{i+1} u_{i} u_{i+1} & \equiv u_{i+1} u_{i+1} u_{i}, \\
u_{i+1} u_{i+2} u_{i+1} u_{i} & \equiv u_{i+1} u_{i+2} u_{i} u_{i+1} .
\end{aligned}
$$

Note that most of these relations do not appear in the list of relations for the algebra of up- and down-operators, as they are implied by the quadratic relations (1)-(5) when the down-operators are included.

It is natural to also consider the subalgebra generated by the down-operators. Let $\mathcal{D}$ be the subalgebra of $\mathcal{U}$ generated by $d_{i}$ for $i \in \mathbf{N}$, and let $I_{\mathcal{D}}=I \cap \mathcal{D}$. The subalgebra of down-operators for Young's lattice is then $\mathcal{D} / I_{\mathcal{D}}$. Recall that with respect to the basis $\mathbf{Y}$, we have $u_{i}^{T}=d_{i}$. Applying this transpose property to the relations in Theorem 15 gives the following characterization of $\mathcal{D} / I_{\mathcal{D}}$.

Theorem 16. The ideal $I_{\mathcal{D}}$ is generated by the following relations:

$$
\begin{aligned}
d_{i} d_{j} & \equiv d_{j} d_{i} \quad \text { for }|i-j| \geqslant 2, \\
d_{i} d_{i+1} d_{i} & \equiv d_{i} d_{i} d_{i+1}, \\
d_{i+1} d_{i} d_{i+1} & \equiv d_{i} d_{i+1} d_{i+1}, \\
d_{i} d_{i+1} d_{i+2} d_{i+1} & \equiv d_{i+1} d_{i} d_{i+2} d_{i+1} .
\end{aligned}
$$

\subsection{Up- and down-operators for a fixed index}

Fix some $1<t \in \mathbf{N}$. Let $\mathcal{B}$ be the subalgebra of $\mathcal{U}$ generated by $u_{t}$ and $d_{t}$, and consider the subalgebra $\mathcal{B} / I_{\mathcal{B}}=\mathcal{B} /\left(I_{\mathcal{U}} \cap \mathcal{B}\right) \subseteq \mathcal{U} / I_{\mathcal{U}}$. We will show that its ideal of relations $I_{\mathcal{B}}$ is generated by

$$
\begin{aligned}
u_{t}^{\eta+1} d_{t}^{\eta} & \equiv u_{t}^{\eta+1} d_{t}^{\eta+1} u_{t} \\
u_{t}^{\eta} d_{t}^{\eta+1} & \equiv d_{t} u_{t}^{\eta+1} d_{t}^{\eta+1}
\end{aligned}
$$

for all $\eta \in \mathbf{N}$. Let $J_{\mathcal{B}}$ be the ideal generated by relations (13) and (14), so that we wish to show $J_{\mathcal{B}}=I_{\mathcal{B}}$.

(When $t=1$, it is straightforward to verify that the only relation between $u_{1}$ and $d_{1}$ is (4), namely $d_{1} u_{1} \equiv i d$, as this relation can be used to rewrite any monomial in the form $u_{1}^{\eta_{1}} d_{1}^{\eta_{2}}$, and all such monomials act independently on $\mathbf{Y}$.) 


\subsubsection{Peaks and valleys}

One convenient way to interpret a word consisting only of the letters $t$ and $\bar{t}$ is as a graph of diagonal steps. More precisely, we construct a graph corresponding to a word $x$ in the following way. Starting at the origin in the plane we read $x$ from right to left. When we encounter a $t$ we take a diagonal step up and to the left by adding $(-1,1)$, and when we encounter a $\bar{t}$ we take a diagonal step down and to the left by adding $(-1,-1)$. One must be careful since we are reading both the word and its graph from right to left.

We call a point of the graph with maximal height a peak and a point with minimal height a valley. (Peaks and valleys need not be unique.) It is straightforward to see that if $(a, b)$ is a peak and $(c, d)$ is a valley, then $\alpha_{t-1}(x)=b$ and $\alpha_{t}(x)=-d$. Also note that if $(e, f)$ is the (leftmost) endpoint of the graph, then $w_{t}(x)=f$. Therefore by Corollary 5 , the action of $x$ on $\mathbf{Y}$ is determined entirely by the heights of its peaks, valleys, and endpoint.

Example 17. The word $x=t^{2} \bar{t}^{4} t^{3}$ has the graph shown below.

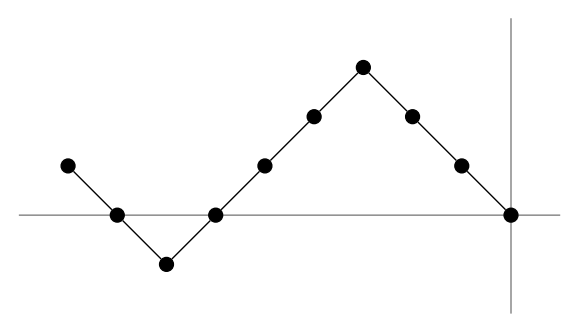

This graph has a peak at $(-3,3)$ and a valley at $(-7,-1)$. Correspondingly, $\alpha_{t-1}(x)=3$ and $\alpha_{t}(x)=1$. The leftmost point of the graph is $(-9,1)$, so $w_{t}(x)=1$.

Note that relations (13) and (14) are not bounded in degree since the only condition on $\eta$ is that it be a nonnegative integer. This differs from the previous algebras that we examined in that the largest degree needed in those cases was 4 (as in the subalgebra of up-operators $\left.\mathcal{U}^{\prime} / I_{\mathcal{U}^{\prime}}\right)$. Indeed, relations of unbounded degree are required due to the following proposition.

Proposition 18. The ideal $I_{\mathcal{B}}$ cannot be generated by elements of bounded degree.

Proof. Suppose for contradiction that the largest degree appearing among the generators of $I_{\mathcal{B}}$ is $h \in \mathbf{N}$. Choose an integer $k>h$, and let $x=t^{k}$ and $y=t^{k} \bar{t}^{k} t^{k}$. Observe that $w(x)=w(y)=(0, \ldots, 0, k, 0, \ldots)$ and $\alpha(x)=\alpha(y)=(0, \ldots, 0, k, 0, \ldots)$, and so $u_{x} \equiv u_{y}$ $\left(\bmod I_{\mathcal{B}}\right)$ by Corollary 5.

Note that in the graph of $x$, there is never a peak occurring to the right of a valley. In other words, if $x=x_{1} \ldots x_{k}$, then there do not exist $i<j$ such that $\left(-i, \alpha_{t-1}(x)\right)$ and $\left(-j,-\alpha_{t}(x)\right)$ appear in the graph of $x$. We will call an instance of a peak occurring to the right of a valley a peak/valley pair. For instance, $x$ has no peak/valley pair but $y$ does, corresponding to the suffix subwords $t^{k}$ and $\bar{t}^{k} t^{k}$, respectively.

We now show that for words $z$ satisfying $\alpha_{t-1}(z)+\alpha_{t}(z)>h$, our degree boundedness assumption implies that the existence of a peak/valley pair is invariant modulo $I_{\mathcal{B}}$. This 
will then lead to an immediate contradiction when applied to $x$ and $y$. Let $u_{m}-u_{m^{\prime}}$ be a generator of $I_{\mathcal{B}}$ of degree at most $h$. It suffices to show that if the word $z=m_{1} m m_{2}$ has a peak/valley pair, then so does $z^{\prime}=m_{1} m^{\prime} m_{2}$.

Since $u_{m} \equiv u_{m^{\prime}}\left(\bmod I_{\mathcal{B}}\right)$, the graphs of $m$ and $m^{\prime}$ must have their peaks, valleys, and endpoints at the same heights. Therefore $z$ has a peak or valley within $m$ if and only if $z^{\prime}$ has a peak or valley within $m^{\prime}$. If $z$ has a peak/valley pair with neither peak nor valley occurring within $m$, then $z^{\prime}$ has a peak/valley pair at the same locations. If at most one of the peak or valley occurs within $m$, say the peak, then the valley must occur within $m_{1}$, so $z^{\prime}$ will have a peak within $m^{\prime}$ and a valley within $m_{1}$ and hence a peak/valley pair. (The other case is similar.) The only remaining possibility is if both the peak and valley occur within $m$ (for they might switch order in $m^{\prime}$ ). However, since $\alpha_{t-1}(z)+\alpha_{t}(z)>h$, the difference in height between the peak and valley is more than $h$, so they cannot both appear within $m$, which has length at most $h$. This completes the proof.

\subsubsection{Proof of relations}

We now prove that relations (13) and (14) suffice. The proofs for the following two propositions are essentially the same as the proofs of the analogous propositions in Section 3.

Proposition 19. The ideal $I_{\mathcal{B}}$ is a binomial ideal.

Proposition 20. The inclusion of ideals $J_{\mathcal{B}} \subseteq I_{\mathcal{B}}$ holds.

As in Section 3, our approach is to construct a standard equivalence class representative $u_{[x]}\left(\operatorname{modulo} I_{\mathcal{B}}\right)$ for every monomial $u_{x}$ and to then show that $u_{x} \equiv u_{[x]}\left(\bmod J_{\mathcal{B}}\right)$.

Definition 21. For any word $x$ in $t$ and $\bar{t}$, define

$$
[x]=t^{w_{t}(x)+\alpha_{t}(x)} \bar{t}^{\alpha_{t-1}(x)+\alpha_{t}(x)} t^{\alpha_{t-1}(x)} .
$$

We say that such a word $[x]$ is the standard representative for $x$, or alternatively that it is in standard form.

Note that all the exponents appearing in $[x]$ are nonnegative: in particular, by the definition of $\alpha_{t}(x)$ we have $\alpha_{t}(x) \geqslant-w_{t}(x)$, and so $w_{t}(x)+\alpha_{t}(x) \geqslant 0$. It is straightforward to check that we have $w(x)=\left(0, \ldots, 0, w_{t}(x), 0, \ldots\right)=w([x])$ and $\alpha(x)=$ $\left(0, \ldots, 0, \alpha_{t-1}(x), \alpha_{t}(x), 0, \ldots\right)=\alpha([x])$, so Corollary 5 implies that $[x]$ is the unique word in standard form such that $u_{x} \equiv u_{[x]}\left(\bmod I_{\mathcal{B}}\right)$.

Proposition 22. Let $x=x_{1} \cdots x_{\ell}$ be a word in $t$ and $\bar{t}$. We have $u_{x} \equiv u_{[x]}\left(\bmod J_{\mathcal{B}}\right)$.

Proof. We prove this by induction on the length of $x$. If $\ell=0$ or if $x=\bar{t}$, then $[x]=x$, so there is nothing to prove. If $x=t$, then $[x]=t \bar{t} t$, and $\mathrm{t} \equiv \mathrm{t} \overline{\mathrm{t}} \mathrm{t}$ by (13) for $\eta=0$.

Now suppose the statement holds for all words shorter than $x$. We have that $u_{x}=$ $u_{x_{1}} u_{y}$ where $y=x_{2} \cdots x_{\ell}$. By induction, $u_{x}=u_{x_{1}} u_{y} \equiv u_{x_{1}} u_{[y]}\left(\bmod J_{\mathcal{B}}\right)$, so we need to show $u_{x_{1}} u_{[y]} \equiv u_{[x]}$. 
If $x_{1}=t$ and $w_{t}(y)<\alpha_{t-1}(y)$, then $w_{t}(x)=w_{t}(y)+1$ while $\alpha(x)=\alpha(y)$. Hence $[x]=t[y]$, so there is nothing to show. Similarly if $x_{1}=\bar{t}$ and $\alpha_{t}(y)=-w_{t}(y)$, then

$$
[x]=\bar{t}^{\alpha_{t-1}(y)+\alpha_{t}(y)+1} t^{\alpha_{t-1}(y)}=\bar{t}[y],
$$

so again there is nothing to show.

Suppose $x_{1}=t$ and $w_{t}(y)=\alpha_{t-1}(y)$. Then $w_{t}(x)=w_{t}(y)+1, \alpha_{t-1}(x)=\alpha_{t-1}(y)+1$, and $\alpha_{t}(x)=\alpha_{t}(y)$. Here the graph of $x$ has a new peak at its leftmost point, so $t[y]$ is not in standard form. Applying (13) with $\eta=w_{t}(y)+\alpha_{t}(y)$ gives

$$
\begin{aligned}
u_{t} u_{[y]} & =\mathrm{t}^{w_{t}(y)+\alpha_{t}(y)+1} \overline{\mathrm{t}}^{w_{t}(y)+\alpha_{t}(y)} \mathrm{t}^{\alpha_{t-1}(y)} \\
& \equiv \mathrm{t}^{w_{t}(y)+\alpha_{t}(y)+1} \overline{\mathrm{t}}^{w_{t}(y)+\alpha_{t}(y)+1} \mathrm{t}^{\alpha_{t-1}(y)+1}=u_{[x]} .
\end{aligned}
$$

Finally, suppose $x_{1}=\bar{t}$ and $\alpha_{t}(y)>-w_{t}(y)$. We then have $w_{t}(x)=w_{t}(y)-1$ and $\alpha(x)=\alpha(y)$. Again $\bar{t}[y]$ is not in standard form since it begins with $\bar{t}$. Note that by definition $\alpha_{t-1}(y) \geqslant w_{t}(y)$, so $\alpha_{t-1}(y)+\alpha_{t}(y) \geqslant w_{t}(y)+\alpha_{t}(y)$. Therefore we can apply (14) with $\eta=w_{t}(y)+\alpha_{t}(y)-1=w_{t}(x)+\alpha_{t}(x)$ to get

$$
\begin{aligned}
& d_{t} u_{[y]}=\overline{\mathrm{t}} \mathrm{t}^{w_{t}(y)+\alpha_{t}(y)} \overline{\mathrm{t}}^{\alpha_{t-1}(y)+\alpha_{t}(y)} \mathrm{t}^{\alpha_{t-1}(y)} \\
& \equiv \mathrm{t}^{w_{t}(y)+\alpha_{t}(y)-1} \overline{\mathrm{t}}^{\alpha_{t-1}(y)+\alpha_{t}(y)} \mathrm{t}^{\alpha_{t-1}(y)}=u_{[x]} .
\end{aligned}
$$

Theorem 23. The ideals $I_{\mathcal{B}}$ and $J_{\mathcal{B}}$ are equal.

Proof. This follows from Propositions 19, 20, and 22.

\subsubsection{Up- and down-operators on finite chains}

Consider again the operators $u_{t}$ and $d_{t}$ for some fixed $t>1$. The action of these operators on $\mathbf{Y}$ splits up as a direct sum of the action on chains $C$, where $C$ is a set of partitions $\lambda$ that have fixed values for $\lambda_{i}^{\prime}$ for all $i \neq t$. The action is then determined entirely by $\rho=\lambda_{t-1}^{\prime}-\lambda_{t+1}^{\prime}$, the difference between the $(t-1)$ st and $(t+1)$ st columns. (Equivalently, $C$ is a chain with $\rho+1$ elements, and $u_{t}$ and $d_{t}$ act as up- and down-operators on this chain.)

Fix $\rho$, and let $I_{\mathcal{C}}$ be the two-sided ideal of $\mathcal{B}$ containing all elements which annihilate $C$, a chain with $\rho+1$ elements. We characterize the algebra $\mathcal{B} / I_{\mathcal{C}}$ by showing that $I_{\mathcal{C}}$ is generated by the following relations:

$$
\begin{aligned}
u_{t}^{\eta+1} d_{t}^{\eta} & \equiv u_{t}^{\eta+1} d_{t}^{\eta+1} u_{t} & & \text { for } 0 \leqslant \eta \leqslant \rho-1, \\
u_{t}^{\eta} d_{t}^{\eta+1} & \equiv d_{t} u_{t}^{\eta+1} d_{t}^{\eta+1} & & \text { for } 0 \leqslant \eta \leqslant \rho-1, \\
u_{t}^{\rho+1} & \equiv 0, & & \\
d_{t}^{\rho+1} & \equiv 0 . & &
\end{aligned}
$$

Let $J_{\mathcal{C}}$ be the ideal generated by relations $(15)-(18)$. We will show that $J_{\mathcal{C}}=I_{\mathcal{C}}$ by exploiting the close relationship between these ideals and $I_{\mathcal{B}}$. 
Theorem 24. The ideals $I_{\mathcal{C}}$ and $J_{\mathcal{C}}$ are equal.

Proof. Recall that $I_{\mathcal{B}}$ is the two-sided ideal of $\mathcal{B}$ containing all elements which annihilate $\mathbf{Y}$. Let $P$ be the two-sided ideal of $\mathcal{B}$ which is generated by relations (17) and (18). It is straightforward to see that $J_{\mathcal{C}}=I_{\mathcal{B}}+P$ (since (13) and (14) for $\eta \geqslant \rho$ are implied by (17) and (18), so we need to show that $I_{\mathcal{C}}=I_{\mathcal{B}}+P$.

The inclusion $I_{\mathcal{B}}+P \subseteq I_{\mathcal{C}}$ holds since both (17) and (18) annihilate $C$. For the reverse direction, note that by Proposition $22, \mathcal{B} / I_{\mathcal{B}}$ has a basis consisting of the standard representatives $u_{[x]}$. A basis element $u_{[x]}$ annihilates $C$ if and only if the power of $\overline{\mathrm{t}}$ appearing in it is larger than $\rho$, which occurs if and only if it lies in $P$. The other basis elements act independently on $C$ as in the proof of Proposition 7. It follows that $I_{\mathcal{C}} \subseteq I_{\mathcal{B}}+P$.

\section{Conclusion}

While the results of this paper and [5] have answered various questions about up- and down-operators, there still remain directions to explore on this subject. For instance, recall that the ideal of relations among the up- and down-operators is generated by relations of bounded degree (in fact, of degree 2), while some subalgebras such as $\mathcal{B} / I_{\mathcal{B}}$ cannot be presented by relations of bounded degree. It would be interesting to determine for which subalgebras this is true. In other words, can one characterize when the generating relations among a subset of operators are bounded versus unbounded in degree?

More generally, it would be interesting to explore these up- and down-operators for posets other than Young's lattice. Let $P$ be a poset with an edge labeling from an index set $I$. We can define up-operators $u_{i}$ for $i \in I$ such that, for $p \in P, u_{i}(p)=q$ if $p \lessdot q$ and the edge between $p$ and $q$ is labeled $i$, and otherwise $u_{i}(p)=0$ if no such $q$ exists. Note that for Young's lattice as considered above, the label between $\lambda$ and $\mu$ where $\lambda \lessdot \mu$ is the column $i$ in which the unique box of $\mu / \lambda$ appears. One can consider the algebras generated by these operators (or the analogously defined $d_{i}$ ) and try to describe their relations for other posets of interest, such as Bruhat order or absolute order on a Coxeter group. (The case of weak order leads to the study of nil-Coxeter algebras [3].) It would also be interesting if it were possible to relate structural properties of these algebras to the structure of the corresponding posets in some way.

\section{References}

[1] Sergey Fomin. Schur operators and Knuth correspondences. J. Combin. Theory Ser. A, 72(2):277-292, 1995.

[2] Sergey Fomin and Curtis Greene. Noncommutative Schur functions and their applications. Discrete Math., 193(1-3):179-200, 1998. Selected papers in honor of Adriano Garsia (Taormina, 1994).

[3] Sergey Fomin and Richard P. Stanley. Schubert polynomials and the nil-Coxeter algebra. Adv. Math., 103(2):196-207, 1994. 
[4] Alain Lascoux and Marcel-P. Schützenberger. Le monö̈de plaxique. In Noncommutative structures in algebra and geometric combinatorics (Naples, 1978), volume 109 of Quad. "Ricerca Sci.", pages 129-156. CNR, Rome, 1981.

[5] Ricky Ini Liu and Christian Smith. The algebra of Schur operators. European J. Combin., 87:103130, 9, 2020.

[6] Joanna Meinel. A plactic algebra action on bosonic particle configurations: The classical case. arXiv:1901.00847.

[7] Richard P. Stanley. Differential posets. J. Amer. Math. Soc., 1(4):919-961, 1988. 